\title{
Study on Several Padé Approximants for Fully lonized Hydrogen Plasmas
}

\author{
Xing-Rong ZHENG ${ }^{1, a^{*}}$, Ji-Hong $\mathrm{LI}^{1, \mathrm{~b}}$, Li-Huan YANG ${ }^{1, \mathrm{c}}$, Yang $\mathrm{LI}^{2, \mathrm{~d}}$ \\ ${ }^{1}$ College of Electrical Engineering, Longdong University, Qingyang 745000, China \\ ${ }^{2}$ School of Physics Science and Technology, Southwest University, Chongqing 400715, China

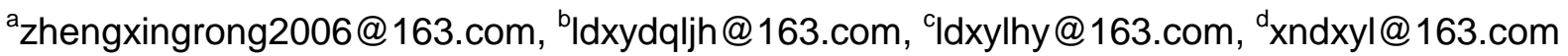 \\ ${ }^{*}$ Corresponding author
}

Keywords: Hydrogen plasma, Fortran program, lonization, Comparison, Padé approximants.

\begin{abstract}
Based on the current proposed three type Padé approximations of fully ionized hydrogen plasmas. Using Fortran program, we calculated the Helmholtz free energy of hydrogen plasmas and made a comparison of these Helmholtz free energy. It is concluded that the dimensionless parameter $f_{e e}{ }^{c}$ of W.Stolzmann's theoretical analysis presents unphysical tendency within $\Gamma_{e}>15$ and $r_{s}<4$, and $f_{i e}{ }^{c}$ gives an unreasonable result within $8.0<\Gamma_{e}<9.1$ and $7.0<r_{s}<8.0$ at low temperature $T=5000 \mathrm{~K}$, but this range of the odd variation and the values of $\Gamma_{e}\left(r_{s}\right)$ will change with the increase of temperature, when the temperature reaches super-high temperature $\left(T>10^{7} \mathrm{~K}\right), f_{i e}{ }^{c}$ almost is applicable to all range of the values of $\Gamma e$ and $r_{s}, f_{e e}{ }^{c}$ is applicable to the values of $0<\Gamma e<0.3$ and $r_{s}>0$. In addition, the dimensionless parameter $f_{i i}{ }^{c}$ of Gilles Chabrier's article and W.Stolzmann's article have a good consistency. Finally, we presented a interpolational formula for hydrogen plasmas, making up for those small odd variation. It demonstrates that the interpolation formula for hydrogen plasmas has a practical application.
\end{abstract}

\section{Introduction}

The Helmholtz free energy of the plasmas are of primary interest in understanding the thermophysical properties at high pressures and temperatures. The primary research of the plasmas is the interaction between the charged particles. The plasmas is the normal state of the matter and a good conductor of electricity, it is of great importance to the development of condensed matter physics, materials science, a planetary physics and weapons physics [1,2]. That is to say, the studies of the ionization of hydrogen at high pressures and temperatures are of practical interest, such as the development of new energy and strategic nuclear weapon $[3,4,5]$. Therefore, many researchers, such as W.Stolzmann, Gilles Chabrier and Setsue Ichimaru, have studied the thermophysical properties of the plasmas, and proposed relevant Padé approximation which provide a completely analytic, accurated description of the thermodynamic quantities of fully ionized electron-ion Coulomb plasmas.

Hydrogen atom have the most simple structure, Therefore, it seems to be an ideal example for the study of the plasma, but hydrogen's complex behavior is beyond previous imagination of scientists at high temperature and pressure. W.Stolzman [6,7,8,9], Gilles Chabrier [10,11] and Setsue Ichimaru $[14,15]$ have studied the interaction of the charged particles using fully ionized plasmas model. W.Stolzmann presented a result of fully ionized plasmas consisting of ions and electrons which are valid in a broad range of temperatures and densities and applicable to arbitrary chemical mixtures by Padé approximants, however, the Padé approximants will be expressed by the dimensionless electronic and ionic plasmas which describe the strength of the Coulomb coupling. In Gilles Chabrier's article, he calculated the thermodynamic of fully ionized electron-ion Coulomb plasmas consisting of different species of pointlike ions and electrons which are encountered in numerous physical and astrophysical situations, and presented a completely analytic formulas for the free energy of electron-ion plasmas, basing on detailed numerical calculations for different ionic species over a wide range of density and temperatures. These Padé formulas provide a useful tool for various application from liquid state theory to dense stellar matter. In Setsue Ichimaru's article, he reviewed the current status of the theoretical developments describing the interparticle correlations in dense plasmas and of the knowledge on the thermodynamic properties and the transport and elementary 
processes, accumulated through theoretical studies since his previous review [15]. He finally presented analytical theories developed for descriptions of static and dynamic correlation in strongly coupled plasmas. We carried on the detailed calculation for all parameters by the above three authors' proposed Padé formulas, and obtained all variable quantities' calculatical results in a broad range of temperatures and densities, including calculatical results of electronic correlation contribution to the Helmholtz free energy $f_{e e}{ }^{c}$ (dimensionless parameter), electronic exchange contribution to the Helmholtz free energy $f_{e e}{ }^{x}$ (dimensionless parameter), ionic correlation contribution to the Helmholtz free energy $f_{i i}{ }^{c}$ (dimensionless parameter), ion-electron correlation contribution to the Helmholtz free energy $f_{i e}{ }^{c}$ (dimensionless parameter) vs. the Coulomb coupling parameter $\Gamma$ and the mean distance between the electrons $r_{s}$ or the mean distance between the ions ri. In this paper, we briefly illustrated advantages of their Padé formulas and local small variations respectively, then we obtained a analytical, detailed and comprehensive result. Finally, we summarized a interpolation formula for fully ionized hydrogen plasmas.

\section{Theoretical Analysis}

The ionization reaction of hydrogen atom will be occurred at high temperatures and pressures, it's ionization processes is

$$
H \Leftrightarrow H^{+}+e
$$

This is to say, hydrogen plasmas consisting of species of $H^{+}$and $e[3,4,5]$. The interaction between the various components are expressed by Padé formulas.

The Helmholtz free energy $F$ of a fully ionized hydrogen consisting of ideal part $F^{i d}$ and Coulomb free energy $F^{\text {coul }}$, which can be written as

$$
F=F^{i d}+F^{\text {coul }} .
$$

where $F^{i d}$ denotes the ideal free energy of ions and electrons, $F^{\text {coul }}$ represents the Coulomb interaction contributions to the free energy. The Coulomb part can decomposed into the following parts.

$$
F^{c o u l}=F_{e e}+F_{i i}+F_{i e} \text {. }
$$

However, W.Stolzmann, Gilles Chabrier and Setsue Ichimaru proposed different Padé formula for the $F_{e e}, F_{i i}, F_{i e}$ [6][10][11][14] which are expressed by the Coulomb coupling parameter $\Gamma_{e}$ and $\Gamma_{i}$, the dimensionless mean distance between the electrons $r_{s}$ and temperature $T$.

$$
\Gamma_{e}=\beta e^{2} / a_{e}, \Gamma_{i}=\beta(Z e)^{2} / a=\Gamma_{e} Z^{5 / 3} .
$$

Where $a_{e}, a$ are the mean distance between the electrons and between the ions, respectively.

$$
a_{e}=\left(\frac{4}{3} \pi n_{e}\right)^{-1 / 3}, a=\left(\frac{4}{3} \pi n_{i o n}\right)^{-1 / 3}=\left(\frac{4}{3} \pi n_{e} / Z\right)^{-1 / 3}=Z^{1 / 3} a_{e} .
$$

where $n_{i o n}$ is the ion density, $n_{e}$ is the electron density, parameter $\beta=1 /\left(4 \pi \varepsilon_{0} k_{B} T\right)$. The temperature and the mean distance between the electrons usually are expressed by the dimensionless $\tau$ and $r_{s}$.

$$
\tau=\frac{k_{B} T}{R y d}, 1 R y d=13.6058 \mathrm{eV}, r_{s}=\frac{a_{e}}{a_{B}}\left(a_{B} \text { is Bohr radius }\right)
$$

\section{Results and Discussion}

In this section, we give a systematic comparison of Padé formulas of the Helmholtz free energy $F_{e e}$, $F_{i i}, F_{i e}$. we calculated all Helmholtz free energy of the fully ionized hydrogen plasmas from low temperature to super-high temperature, but mainly listed four typical isotherms of the Helmholtz free 
energy $f$ (dimensionless parameter) vs. the Coulomb coupling parameter $\Gamma$ ( $\Gamma_{e}=\Gamma_{i}$, for hydrogen) and the mean distance between the electrons $r_{s}\left(r_{s=} r_{i}\right.$, for hydrogen $)$.

\section{Electron-electron Interaction Contribution to the Helmholtz Free Energy $F_{e e}\left(\Gamma_{e}, r_{s}, T\right)$}

Electron-electron interaction contribution to the Helmholtz free energy $F_{e e}$ of W.Stolzmann's article is expressed by the dimensionless electronic plasmas parameters $\left(\Gamma_{e}, r_{s}, T\right)$, which describe the strength of the Coulomb coupling $[8,14]$

$$
\begin{aligned}
& F_{e e}=F_{e e}^{c}+F_{e e}^{x} . \\
& f_{e e}^{c}=\frac{F_{e e}^{c}}{N_{e} k_{B} T}=-\frac{a_{0} \Gamma_{e}^{3 / 2}-a_{2} \Gamma_{e}^{6}\left[\varepsilon_{c}\left(R_{s}\right)+\Delta \varepsilon_{c}\left(R_{s}, \tau\right)\right] / \tau}{1+a_{1} a_{3} \Gamma_{e}^{3 / 2}+a_{2} \Gamma_{e}^{6}}, \\
& f_{e e}^{x}=\frac{F_{e e}^{x}}{N_{e} k_{B} T}=-\frac{2 e^{2}}{\Lambda_{e}^{4} n_{e} k_{B} T} \int_{-\infty}^{\Psi} d \Psi^{\prime} I_{-1 / 2}^{2}\left(\Psi^{\prime}\right) .
\end{aligned}
$$

where $\Lambda_{e}$ is the thermal De Broglie-wavelength $\left(\Lambda_{e}=2 \pi \hbar \sqrt{2 m_{e} \pi k_{B} T}\right)$, other parameters are given in Ref. [6].

While electron-electron interaction contribution to the Helmholtz free energy $f_{e x}$ of Setsue Ichimaru's article[14] is given by

$$
\begin{aligned}
-f_{e x}= & \frac{F_{e x}}{N k_{B} T}=\frac{c}{e} \Gamma+\frac{2}{e}\left(b-\frac{c d}{e}\right) \Gamma^{1 / 2}+\frac{1}{e}\left[\left(a-\frac{c}{e}\right)-\frac{d}{e}\left(b-\frac{c d}{e}\right)\right] \ln \left|e \Gamma+d \Gamma^{1 / 2}+1\right| \\
& -\frac{2}{e\left(4 e-d^{2}\right)^{1 / 2}}\left[d\left(a-\frac{c}{e}\right)+\left(2-\frac{d^{2}}{e}\right)\left(b-\frac{c d}{e}\right)\right] \times\left[\tan ^{-1} \frac{2 e \Gamma^{1 / 2}+d}{\left(4 e-d^{2}\right)^{1 / 2}}-\tan ^{-1} \frac{d}{\left(4 e-d^{2}\right)^{1 / 2}}\right]
\end{aligned}
$$

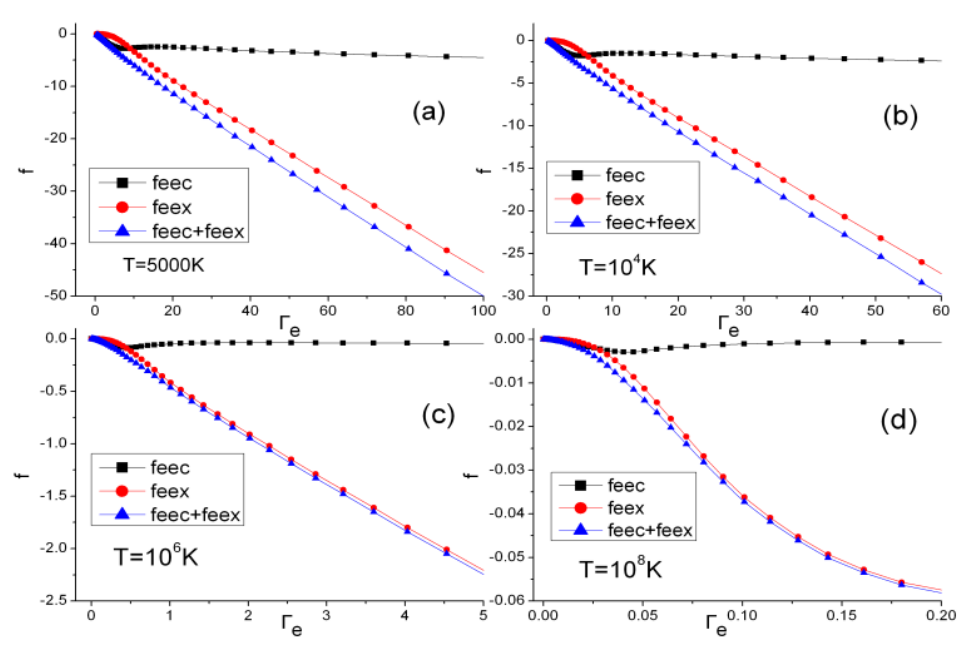

Fig.1 The Helmholtz free energy $f_{e e}{ }^{c}, f_{e e}{ }^{x}, f_{e e}{ }^{c}+f_{e e}{ }^{x}$ vs. the electron coupling parameter $\Gamma_{e}$ for four isotherms of hydrogen plasma from W.Stolzmann's theoretical analysis ${ }^{[6]}$ at the temperatures values of $T=5000 \mathrm{~K}, 10^{4} \mathrm{~K}, 10^{6} \mathrm{~K}, 10^{8} \mathrm{~K}$

Based on several proposed Padé formulas of W.Stolzmann's theoretical analysis [6], using calculational results, we made a comparison for four isotherms with the Helmholtz free energy $f_{e e}{ }^{c}$, $f_{e e}{ }^{x}, f_{e e}{ }^{c}+f_{e e}{ }^{x}$, the electron coupling parameter $\Gamma_{e}$ in a broad range of temperature, as shown in Fig. 1 . We find that the value of $f_{e e}{ }^{c}$ is small, while the value of $f_{e e}{ }^{x}$ is very big and mainly play an important role at the strongly coupling limit, but with the decrease of the value of the electron coupling parameter $\Gamma_{e}$ and the increase of the value of the mean distance between the electrons $r_{s}, f_{e e}{ }^{c}$ and $f_{e e}{ }^{x}$ tend to get balance. Such as at low temperatures $T=5000 \mathrm{~K}, f_{e e}{ }^{c}$ can be ignored comparing with $f_{e e}{ }^{x}$ within $\Gamma_{e}>15$ and $r_{s}<4$, but the difference between the value of $f_{e e}{ }^{c}$ and $f_{e e}{ }^{x}$ get bigger with the increase of $\Gamma_{e}$ at high temperatures. Detailed quantitative analysis is as following, as shown in picture (b) $\left(T=10^{4} \mathrm{~K}\right)$, when $\Gamma_{e}>6\left(r_{s}<5\right)$, the value of $f_{e e}{ }^{c}$ much smaller than the value of $f_{e e}{ }^{x}$, so $f_{e e}{ }^{c}$ can be 
ignored, the curves of $f_{e e}{ }^{x}$ and $f_{e e}{ }^{c}+f_{e e}{ }^{x}$ get very close, but with the increases of temperature, the value of this range also is being changed. As shown in the picture (c), this range becomes $\Gamma_{e}>0.4\left(r_{s}<0.6\right)$. When the temperature reaches the values $T=10^{8} \mathrm{~K}$, as shown in the picture (d), the electron coupling parameter approximately reaches the values $\Gamma_{e}>0.025$, while the mean distance between the electrons approximately reaches the values $r_{s}<0.08$.

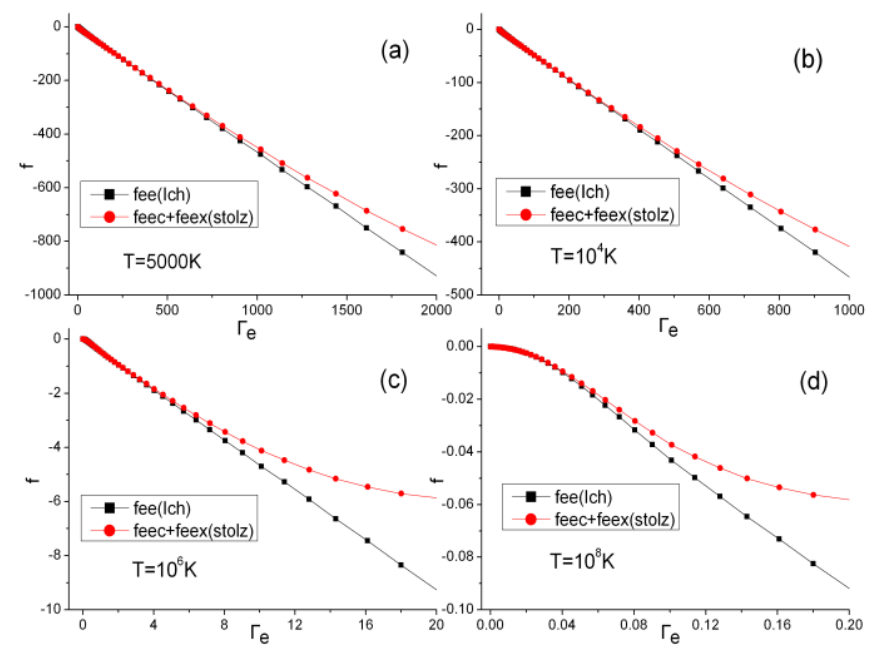

Fig.2 The Helmholtz free energy fee of Setsue Ichimaru's article and $f_{e e}{ }^{c}+f_{e e}{ }^{x}$ of W.Stolzmann's article vs. the electron coupling parameter $\Gamma_{e}$ at the temperatures values of $T=5000 \mathrm{~K}, 10^{4} \mathrm{~K}, 10^{6} \mathrm{~K}$, $10^{8} \mathrm{~K}$. The filled circles refers to the Padé approximant of $f_{e e}{ }^{c}+f_{e e}{ }^{x}$ from Stolzmann [6], the filled squares show the Padé approximant of $f_{e e}$ from Ichimaru [10].

We also made a comparison between $f_{e e}$ of Setsue Ichimaru's article [14] and $f_{e e}{ }^{c}+f_{e e}{ }^{x}$ of W.Stolzmann's article [6], as shown in Fig.2. By calculating, we obtained that two curves of $f_{e e}$ and $f_{e e}{ }^{c}+f_{e e}{ }^{x}$ have very good consistency at the small of $\Gamma_{e}$ and the big of $r_{s}$ at the temperatures ranging from $T=5000 \mathrm{~K}$ to $T=10^{8} \mathrm{~K}$, but we will adopt $f_{e e}$ of Setsue Ichimaru's article [14] if calculate the value of $f_{e e}$, because it can be more accurately express the value of $f_{e e}$ while the value of $f_{e e}{ }^{c}+f_{e e}{ }^{x}$ gives an unreasonable result at the big region of $\Gamma_{e}$ and the small region of $r_{s}$. By comparison, we can see that the unreasonable result of electronic correlation contribution to the Helmholtz free energy $f_{e e}{ }^{c}$ cann't be neglected if calculate $f_{e e}{ }^{c}+f_{e e}{ }^{x}$ (equal to $f_{e e}$ ) at the big region of $\Gamma_{e}$ and the small region of $r_{s}$, and the value of $f_{e e}{ }^{c}$ almost has effects on $f_{e e}$ or $f_{e e}{ }^{c}+f_{e e}{ }^{x}$ at the big region of $\Gamma_{e}$ and the small region of $r_{s}$. This also shows that $f_{e e}{ }^{c}$ has effects on $f_{e e}{ }^{x}$ within a certain some range of $\Gamma_{e}$ and $r_{s}$.

\section{Ion-ion Correlation Contribution to the Helmholtz Free Energy $F_{i i}{ }^{c}\left(\Gamma_{e}\right)$}

Ion-ion correlation contribution to the Helmholtz free energy $F_{i i}{ }^{c}$ of W.Stolzmann's article [6] is given by

$$
f_{i i}^{c}=\frac{F_{i i}^{c}}{N_{i} k_{B} T}=-\frac{b_{0} \Gamma_{i}^{3 / 2}+b_{2} \Gamma_{i}^{9 / 2} \varepsilon_{i i}}{1+b_{1} \Gamma_{i}^{3 / 2}+b_{2} \Gamma_{i}^{9 / 2}} .
$$

while ion-ion correlation contribution to the Helmholtz free energy $F_{i i}{ }^{c}$ of Gilles Chabrier's the first article [10] is given by

$$
\begin{aligned}
f_{i i}^{c}\left(\Gamma_{i}\right)= & A_{1}\left[\sqrt{\Gamma_{i}\left(A_{2}+\Gamma_{i}\right)}-A_{2} \ln \left(\sqrt{\Gamma_{i} / A_{2}}+\sqrt{1+\Gamma_{i} / A_{2}}\right)\right] . \\
& +2 A_{3}\left[\sqrt{\Gamma_{i}}-\arctan \left(\sqrt{\Gamma_{i}}\right)\right]
\end{aligned}
$$

Using the above Padé formulas, we mainly introduced ionic correlation contribution to the Helmholtz free energy $f_{i i}{ }^{c}$ of Gilles Chabrier's two article [10], and compared with the Padé approximant of $f_{i i}^{c}$ from Stolzmann's article [6]. As shown in Fig.3, we give a comparison between $f_{i i}{ }^{c}$ of Gilles Chabrier's articles [10] and $f_{i i}{ }^{c}$ of W.Stolzmann's article [6]. It is concluded that the ionic correlation contribution to the Helmholtz free energy $f_{i i}{ }^{c}$ of W.Stolzmann's article and $f_{i i}{ }^{c}$ of Gilles Chabrier's articles[10] have a very good Consistency and the curve of them almost completely close 
at different temperatures ranging from $T=5000 \mathrm{~K}$ to $T=10^{8} \mathrm{~K}$. Therefore, $f_{i i}^{c}$ of Gilles Chabrier's articles and W.Stolzmann's article are very reasonable for ion-ion interaction.

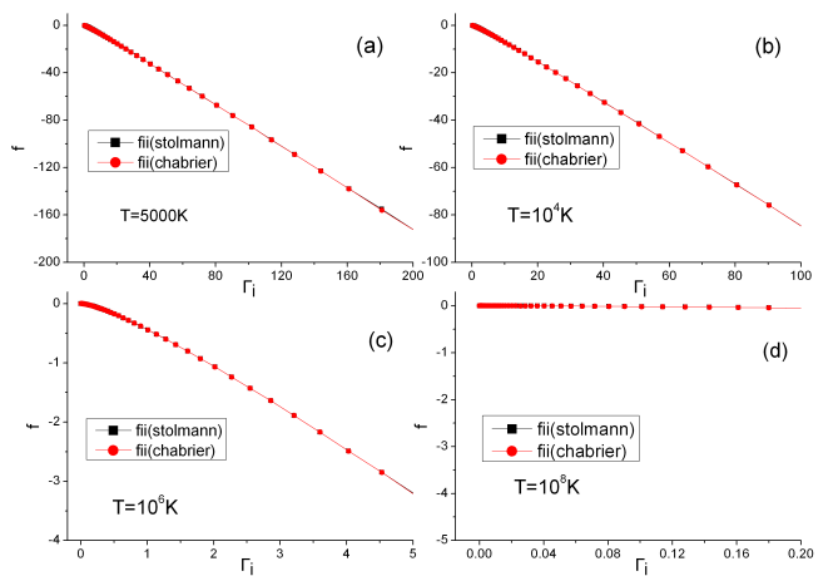

Fig. 3 The ionic correlation contribution to the Helmholtz free energy $f_{i i}{ }^{c}$ vs. the ion coupling parameter $\Gamma_{i}$ by with the datas given by Gilles Chabrier's theoretical analysis and W.Stolzmann's article for different temperatures ranging from $T=5000 \mathrm{~K}$ to $T=10^{8} \mathrm{~K}$. The filled squares refers to the Padé approximant of $f_{i i}{ }^{c}$ from Stolzmann's articles [6], the filled circles show the Padé approximant of $f_{i i}{ }^{c}$ from Gilles Chabrier's articles [10].

\section{Ion-electron Correlation Contribution to the Helmholtz Free Energy $\boldsymbol{F}_{i e}{ }^{c}\left(\boldsymbol{\Gamma}_{e}, \boldsymbol{r}_{s}\right)$}

Ion-electron correlation contribution to the Helmholtz free energy $F_{i e}{ }^{c}$ of W.Stolzmann's article [6] is expressed by

$$
f_{i e}^{c}=\frac{F_{i e}^{c}}{N_{i} k_{B} T}=-\frac{c_{0} \Gamma_{i}^{3 / 2}+c_{2} \Gamma_{e}^{9 / 2} \varepsilon_{i e}}{1+c_{1} \Gamma_{i}^{3 / 2}+c_{2} \Gamma_{e}^{9 / 2}+2 c_{4} \Gamma_{i}^{3 / 2} \ln \left[1+\left(c_{5} / \Gamma_{i}^{3}\right)^{1 / 2}\right]} \cdot
$$

Ion-electron correlation contribution to the Helmholtz free energy $F_{i e}{ }^{c}$ of Gilles Chabrier's the article $[10,11]$ is expressed by

$$
f_{i e}^{c}=\frac{F_{i e}}{N_{i} k_{B} T}=-\Gamma_{e} \frac{c_{D H} \sqrt{\Gamma_{e}}+c_{T F} a \Gamma_{e}^{v} g_{1} h_{1}}{1+\left[b \sqrt{\Gamma_{e}}+a g_{2} \Gamma_{e}^{v} / r_{s}\right] h_{2}} .
$$

As shown in Fig.4, by calculating, we obtained the following conclusion by above Padé formulas. There are three major issues. First, we made a comparison between ion-electron correlation contribution to the Helmholtz free energy $f_{i e}{ }^{c}$ of Gilles Chabrier's article [10] and W.Stolzmann's article [6] at different temperatures of $T=5000 \mathrm{~K}, 10^{4} \mathrm{~K}, 10^{6} \mathrm{~K}, 10^{8} \mathrm{~K}$. As shown in Fig.4, there are differences between them in small region of $\Gamma_{e}$ and $r_{s}$ at different temperatures ranging from $T=5000 \mathrm{~K}$ to $T=10^{8} \mathrm{~K}$, but with increase of the temperature, this region of difference get smaller and smaller, however, they have very good agreement at smaller region of $\Gamma_{e}$ and bigger region of $r_{s}$ at all temperatures. Second, it showed that ion-electron correlation contribution to the Helmholtz free energy $f_{i e}{ }^{c}$ vs. the electron coupling parameter $\Gamma_{e}$ by with the datas given by Gilles Chabrier's theoretical analysis[10] for different temperatures ranging from $T=5000 \mathrm{~K}$ to $T=10^{8} \mathrm{~K}$. As shown in Fig.4, our calculation is suitable for the value of $\Gamma_{e}>0$ and $r_{s}>0$, the curve of $f_{i e}{ }^{c}$ doesn't present any unreasonable result and is very smooth in all region of $\Gamma_{e}$ and $r_{s}$ at different temperatures of $T=5000 \mathrm{~K}$, $10^{4} \mathrm{~K}, 10^{6} \mathrm{~K}, 10^{8} \mathrm{~K}$. Three, it described ion-electron correlation contribution to the Helmholtz free energy $f_{i e}{ }^{c}$ vs. the electron coupling parameter $\Gamma_{e}$ by with the datas given by W.Stolzmann's theoretical analysis for different temperatures ranging from $T=5000 \mathrm{~K}$ to $T=10^{8} \mathrm{~K}$. As shown in Fig.4, $f_{i e}{ }^{c}$ gives an unreasonable result within $8.0<\Gamma_{e}<9.1$ and $7.0<r_{s}<8.0$ at low temperature $T=5000 \mathrm{~K}$, but this range of the unphysical tendency and the values of $r_{s}$ get small with the increase of temperature, when the temperature reaches the values $T>10^{5} \mathrm{~K}$, the unphysical tendency disappears, $f_{i e}{ }^{c}$ almost is applicable to all range of $\Gamma_{e}$ and $r_{s}$, and the curve of $f_{i e}{ }^{c}$ is very smooth at all the region of $\Gamma_{e}$ and $r_{s}$. 


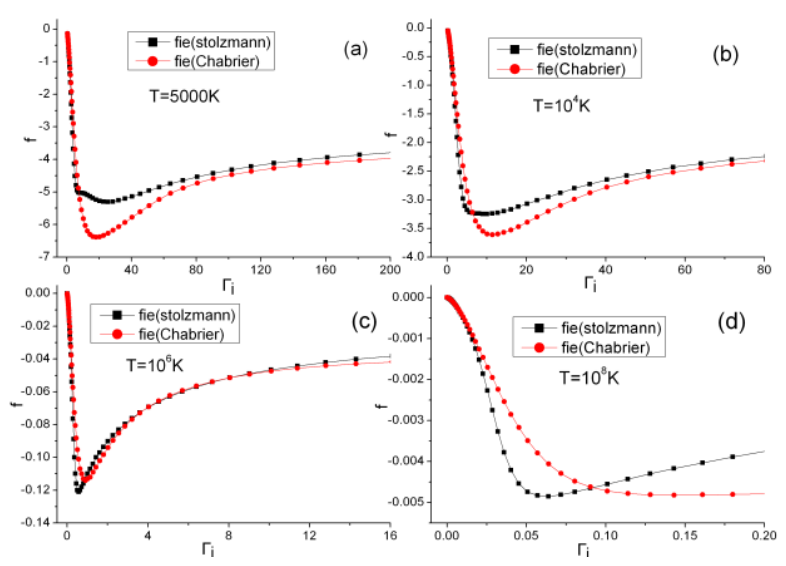

Fig.4 The ion-electron correlation contribution to the Helmholtz free energy $f_{i e}{ }^{c}$ of Gilles Chabrier's article[10] and $f_{i e}{ }^{c}$ of W.Stolzmann's article [6] vs. the electron coupling parameter $\Gamma_{e}$ for different temperatures ranging from $T=5000 \mathrm{~K}$ to $T=10^{8} \mathrm{~K}$. The filled circles refers to the Padé approximant of $f_{i e}{ }^{c}$ from Stolzmann's articles [6], the filled squares show the Padé approximant of $f_{i e}{ }^{c}$ from Chabrier's articles [10].

From the above calculative results of several Padé approximants (as shown in Fig.1 4), We can see that their Padé formulas present odd variation in some regions. Their Padé formulas have its own advantages, but there are some drawwbacks in small regions (it were pointed out in the above figure). Therefore, we obtained a interpolation formula in the following conclusion, making up the local shortcomings.

\section{Conclusion}

We have calculated the Helmholtz free energy of the fully ionized electron-ion Coulomb plasmas of W.Stolzmann, Gilles Chabrier and Setsue Ichimaru's theoretical analysis by using Padé approximants. These approximants is realistic in various conditions at a wide range of temperatures or densities. Furthermore, these approximants were applicable to any chemical mixtures. Finally, We found out some regions of odd variation by calculation, and obtained their respective advantages and disadvantages by compare with several the author's theoretical analysis. Therefore, we used the following interpolational formula if calculating the excess free energy of hydrogen plasmas.

$$
F_{e x}=F_{e e}+F_{i i}^{c}+F_{i e}^{c},
$$

where $F_{e e}$ denotes the interaction between electronic and electronic, coming from Setsue Ichimaru's article[14], and it's dimensionless is expressed as

$$
\begin{aligned}
-f_{e x}= & -\frac{F_{e e}}{N_{e} k_{B} T}=\frac{c}{e} \Gamma+\frac{2}{e}\left(b-\frac{c d}{e}\right) \Gamma^{1 / 2}+\frac{1}{e}\left[\left(a-\frac{c}{e}\right)-\frac{d}{e}\left(b-\frac{c d}{e}\right)\right] \ln \left|e \Gamma+d \Gamma^{1 / 2}+1\right| \\
& -\frac{2}{e\left(4 e-d^{2}\right)^{1 / 2}}\left[d\left(a-\frac{c}{e}\right)+\left(2-\frac{d^{2}}{e}\right)\left(b-\frac{c d}{e}\right)\right] \times\left[\tan ^{-1} \frac{2 e \Gamma^{1 / 2}+d}{\left(4 e-d^{2}\right)^{1 / 2}}-\tan ^{-1} \frac{d}{\left(4 e-d^{2}\right)^{1 / 2}}\right]
\end{aligned}
$$

$F_{e e}{ }^{c}$ denotes the interaction between ion and ion, coming from W.Stolzmann's article, and it's dimensionless is expressed as

$$
f_{i i}^{c}=\frac{F_{i i}^{c}}{N_{i} k_{B} T}=-\frac{b_{0} \Gamma_{i}^{3 / 2}+b_{2} \Gamma_{i}^{9 / 2} \varepsilon_{i i}}{1+b_{1} \Gamma_{i}^{3 / 2}+b_{2} \Gamma_{i}^{9 / 2}}
$$

$F_{i e}{ }^{c}$ denotes the interaction between ion and electronic, coming from Gilles Chabrier's article, and it's dimensionless is expressed as

$$
f_{i e}^{c}=\frac{F_{i e}}{N_{i} k_{B} T}=-\Gamma_{e} \frac{c_{D H} \sqrt{\Gamma_{e}}+c_{T F} a \Gamma_{e}^{v} g_{1} h_{1}}{1+\left[b \sqrt{\Gamma_{e}}+a g_{2} \Gamma_{e}^{v} / r_{s}\right] h_{2}} .
$$

Finally, we give several curves of the coulomb interaction contribution to the Helmholtz free energy $f_{e e}{ }^{c}, f_{i e}{ }^{c}, f_{i e}{ }^{c}$ between charged particles of fully ionized hydrogen plasma at low temperature $T=5000 \mathrm{~K}$. 


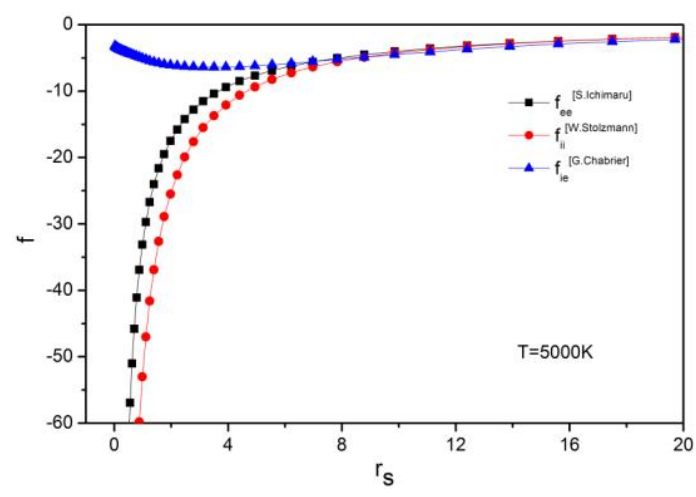

Fig. 5 The coulomb interaction contribution to the Helmholtz free energy between charged particles of fully ionized hydrogen plasma at low temperature $T=5000 \mathrm{~K}$.

\section{Acknowledgement}

In this paper, the research was sponsored by the Nature Science Foundation of Qingyang City, Gansu Province (Project No. ZJ201306).

\section{References}

[1]Nellis W J, Ross M, Holmes N C, SCHience, 269(1995) 1249.

[2]Ebeling W, Forster A, Fortov V E and Grynaznov V K, Thermophysical properties of hot dense plasmas, ed. B. G. Teuber Verlagsgesell SCHhaft Stuttgart Leipzig, 1991 p7.

[3]Saumon D and Chabrier G, Phys Rev A., 1991445122.

[4]Saumon D and Chabrier G, Phys Rev Lett.,1989 622397.

[5]Saumon D and Chabrier G, Phys Rev A.,1992 462084.

[6]Stolzmann W and Blöcker T, Physics Letters A., 221 (1996) 99-103.

[7]Stolzmann W and Blöcker T, Astron.Astrophys., 361 (2000) 1152.

[8]Stolzmann W and Blöcker T, Astron. Astrophys., 314 (1996) 1024-1040.

[9]Stolzmann W and Ebeling W, Physics Letters A., 248 (1998) 242-246.

[10]G.Chabrier and A.Y.Potekhin, Phys.Rev.E., 58 (1998) 4941-4949.

[11]G.Chabrier and A.Y.Potekhin, Phys.Rev.E., 62 (2000) 8554-8563.

[12]G.Chabrier and J.,Phys.(Pairs), 51 (1990) 1607.

[13]M.D.Jones and D.M.Ceperley, Phys.Rev.Lett., 76 (1996) 4572.

[14]Ichimaru.S, Iyetomi.H, Tanaka.S, Phys.Rep., 149(1987) 91-205.

[15]Ichimaru.S, Rev. Mod.Phys., 54(1982) 1017.

[16]F.Perrot and M.W.C.Dharma-wardana, Phys.Rev.A., 30 (1984) 2619.

[17]W.Ebeling,Contrib.Plasmas Phys., 30 (1990) 553.

[18]W.Ebeling and W.Richert, Phys.stat.sol.(b),128 (1990) 467.

[19]Ichimaru.S,.(ed.), 1990, Strongly Coupled Plasmas Physics, North-Holland Delta Series, Amesterdam.

[20]Ichimaru.S, 1994, Statistical Plasmas Physics, Vol. II : Condensed Plasmas, Addison-Wesley Publishing Company, Reading, Massachusetts.

[21]SCHhwarv V, Juranek H, Redmer R, Chem.Phys.,2005 71990.

[22]Ternovoi V Ya, Kvitov S V, Pyalling A A et al, JETP Lett., 79(2004) 8.

[23]Ternovoi V Ya, Filimonov A S,Pyalling A A et al 2001 Shock Compression of Condensed Matter (New York: Melville) 107. 